\title{
3D RECONSTRUCTION AND SLM SURVEY FOR DENTAL IMPLANTS
}

\author{
ZHOU HONGFU* and FAN QIN ${ }^{\dagger},+$ \\ *School of Mechanical and Automobile Engineering \\ South China University of Technology \\ 510641 Guangzhou, China \\ ${ }^{\dagger}$ School of Machinery and Automation \\ Wuhan University of Science and Technology \\ 430081 Wuhan, China \\ \$anqin@wust.edu.cn
}

Received 11 October 2015

Revised 13 July 2016

Accepted 7 December 2016

Published 21 April 2017

\begin{abstract}
This research studies an integrated dental 3D reconstruction scanning method which combines impression model scanning, intraoral camera scanning and CT scanning. In the dental integrated scanning, tooth root data is acquired from CT DICOM data and 3D reconstruction is processed by Mimics ${ }^{\circledR}$ software developed from Materialise. The tooth crown and the tooth bridge $3 \mathrm{D}$ data are obtained by scanning the tooth impression model or scanning with an intraoral scanner. Also, it surveys that the dental prosthesis are fabricated with traditional methods and printed with Select Laser Melting (SLM) technology. In the research, it presents that SLM additive manufacturing (AM) method can fabricate customized dental prosthesis with high dimensional accuracy.
\end{abstract}

Keywords: Dental implant design; tooth scanning; dental additive manufacturing; SLM printing; dental prosthesis.

\section{Introduction}

A dental implant is a prosthesis inserted into a jaw bone to anchor an artificial tooth crown or denture. The dental implant restores the patient's mastication function. Dental implants are a strategy for tooth replacement. The research areas for dental implant application include dental 3D design, dental 3D scanning, dental material, and dental fabricating.

\$Corresponding author.

This is an Open Access article published by World Scientific Publishing Company. It is distributed under the terms of the Creative Commons Attribution 4.0 (CC-BY) License. Further distribution of this work is permitted, provided the original work is properly cited. 
The 3D dental implant design includes the tooth crown design and the tooth alveolar bone design in reconstructing complex dental models. For the root implant, it develops the root-forming endosseous dental implants, which is a fixture that is surgically embedded in the patient's bone to support the dental prosthetics, and endosseous dental abutments.

Dental scanning is a reverse engineering method, wherein a scanner machine scans an impression model, ${ }^{1}$ or an intraoral scanner directly digitizes the tooth crown. ${ }^{2}$ In both methods, these scanners collect the bone data about tooth crown and the occlusal correction. Computed tomography (CT) collects data for tooth root fixture by using CT cloudy data to rebuild the root surfaces. ${ }^{3}$ The dental CT image processing and the dental $3 \mathrm{D}$ reconstruction help the clinician to place the dental implant precisely.

Previous methods to fabricate the dental prosthesis have included casting, computer aid design and computer aid manufacture (CAD/CAM) method, which uses subtractive manufacturing with a CNC machine to cut the material. The traditional method uses the CAD/CAM technology to fabricate a supported maxillary implant and a removable complete dental prosthesis. Recently, this method uses Select Laser Melting (SLM), an additive manufacturing (AM) method that builds a component by a layer-by-layer method from a CAD model, to fabricate the crown, bridge, and tooth root from the $3 \mathrm{D}$ dental scanning data. ${ }^{4-6}$ The AM method uses a laser to customize the dental features and the dental prostheses. Also, the AM processes can create the fine detail and complex internal geometries for dental prostheses. ${ }^{7}$

With advances in dental implant technology, dental implant material has grown to include metallic, ceramic, and polymeric biomaterials. The dental implant metals include cobalt chromium alloy ( $\mathrm{Co}-\mathrm{Cr}$ ), and titanium alloy (Ti6Al4V). Titanium is used extensively in dentistry, especially in denture framework and base plates because of its properties such as low specific weight, low modulus of elasticity, high strength-to-weight ratio, high durability, high corrosion and oxidation resistance, and high biocompatibility, bioactive and osseointegration. ${ }^{8}$ Also, comparing with the commercially pure titanium material, the Ti6Al4V has higher strength and fatigue resistance. Other materials, such as zirconium, might be used to fabricate implants in the future. ${ }^{9}$ The bioactive material should enhance or stimulate the new bone formation and promote the bone-implant attachment. ${ }^{10}$

Porous surfaces on dental implants are created by sintering or fusing the material together, ${ }^{11}$ or by using AM technology to add pores in design, or by the foamed metal method. Porosity has been proven to improve clinical success by increasing the surface contact area. Also, pores provide favorable mechanical conditions for bone growth. ${ }^{12}$

\section{Dental 3D Data Scanning}

The dental implant requires the information on the complete tooth profile and alveolar bone to position the implant. The tooth crown and tooth bridge scanning includes the impression model scanning, the intraoral scanning, ${ }^{13,14}$ and CT scanning. 


\subsection{Indirect scanning method}

A clinician first makes a dental impression model, then scans the model, and finally acquires tooth crown $3 \mathrm{D}$ data via reverse engineering. The impression model recovers the tooth crown and occlusion 3D data used to fabricate the tooth crown and denture. Also, for impression model scanning, the scanner can scan the single to multi-unit implant bridges, over-dentures, individualized abutments, cast model impressions, etc.

Impression models are necessary for accurate diagnoses and treatment planning, and for monitoring treatment progress and routine practice in clinical specialties.

Figure 1(a) shows a dental impression model where the fabricating materials, such as plaster, wax, etc., can be used. Figure 1(b) shows the fabricating processing wherein the material is poured into the mouth bite model to generate an impression

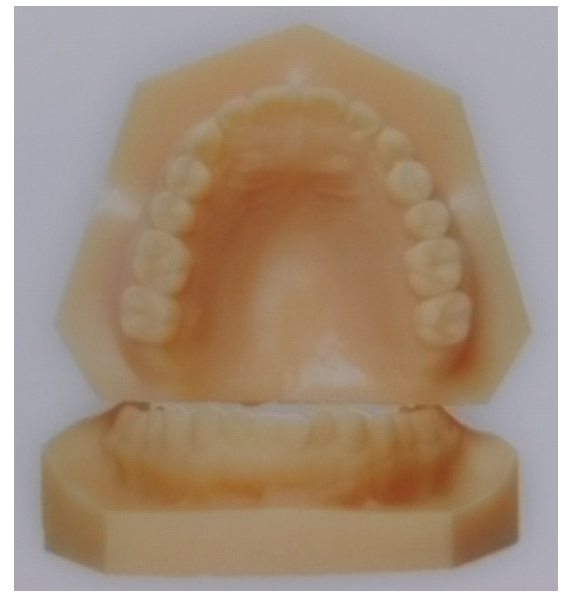

(a)

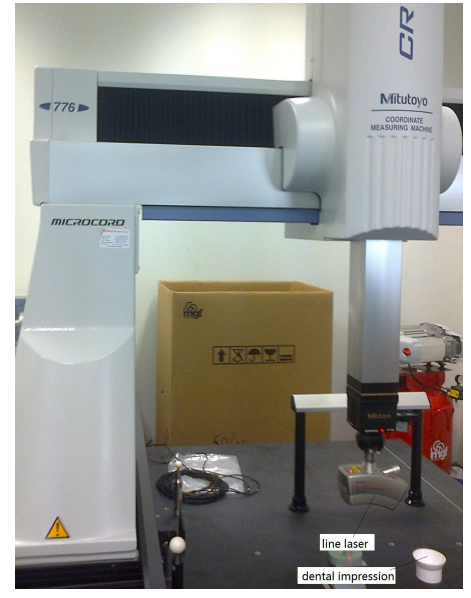

(b)

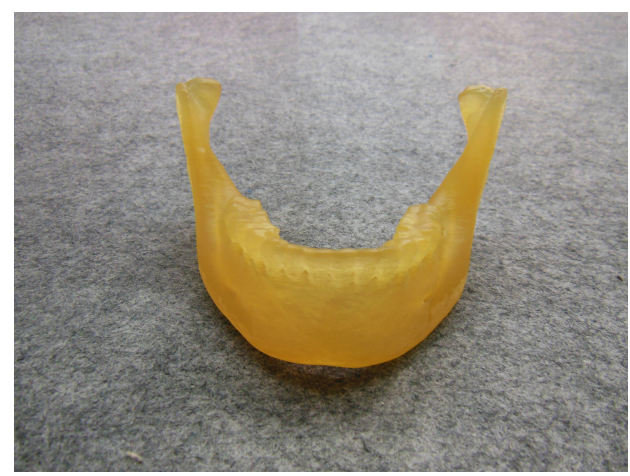

(c)

Fig. 1. (Color online) Scanning dental impression model: (a) Dental impression model, (b) Dental impression Scanning and (c) Scanning dental printing. 
model. Then, the device scans the dental impression model to acquire the dental crown CAD data directly. However, plaster models only provide crown and occlusal data. Finally, the model is printed by AM printing using the generated standard triangular language (STL) data, as shown in Fig. 1(c). The impression model may be replaced by an intraoral scanner, because the intraoral scanner is faster and more efficient.

\subsection{Intraoral scanning}

For direct imaging, an intraoral camera takes a photo from inside the patient's oral cavity and acquires the tooth's 3D data by image processing, as shown in Fig. 2.

Figure 3 shows the result of an intraoral scanner using the mouth dental image to obtain $3 \mathrm{D}$ data via image processing and printing.

The 3D data reconstruction from CT scanning point cloudy data shows in Fig. 4.

As shown in Fig. 5, dental data is obtained from scanning, which is used to print the dental model via a SLA.

\subsection{CT scanning}

The dental impression model and intraoral scanner image only record oral data without recording the tooth root information. The tooth root and crown designs are based on x-ray photos and CT jaw scans. The x-ray photo is the radiation pass through the body to appear on an x-ray film, and it can record the dental root information, but it is difficulty to use in the 3D rebuilding, as shown in Fig. 6. The CT scanning is for $3 \mathrm{D}$ reconstruction, where the $\mathrm{CT}$ data is processed to generate

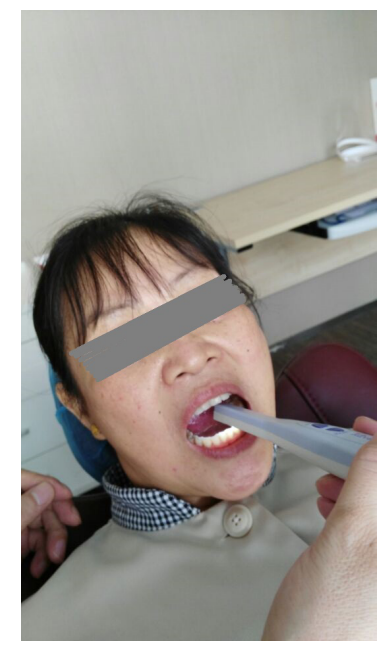

(a)

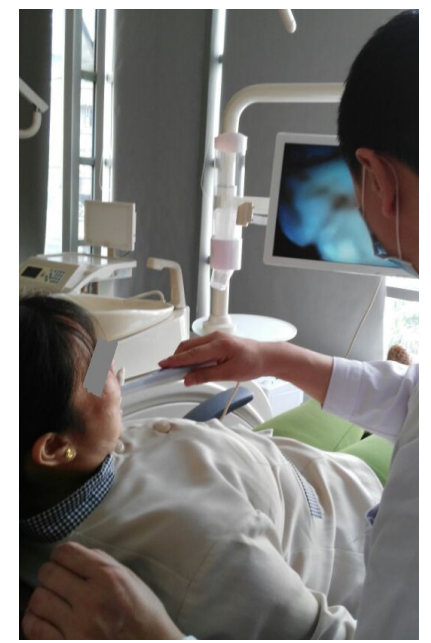

(b)

Fig. 2. (Color online) Intraoral scanner: (a) Intraoral camera, (b) intraoral scanning, (c) image of mouth inside scanning and (d) image of mouth outside scanning. 


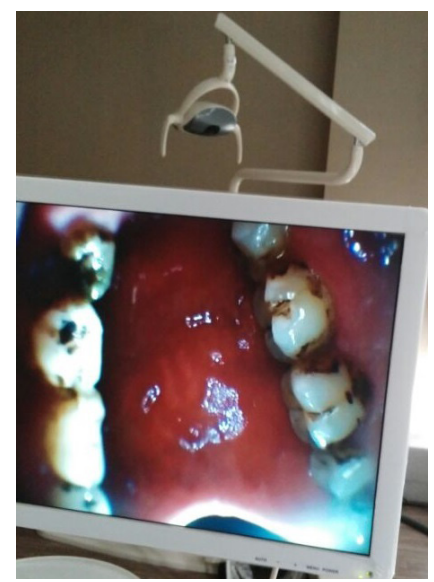

(c)

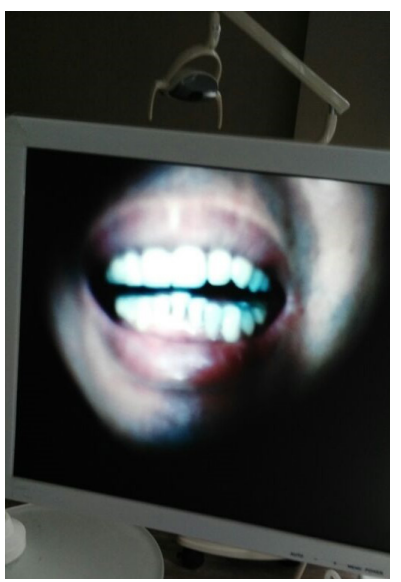

(d)

Fig. 2. (Continued)

into 3D dental cloudy point data and STL data file. ${ }^{15}$ The CT scan data is saved in a Digital Imaging and Communications (DICOM) format. The DICOM is a protocol that describes the formatting and exchanging of image-related information. ${ }^{16}$ Dental DICOM data comes from a Cone Beam CT or a Medical CT. However, the Medical CT may limit the applications in routine diagnosis due to the amount of

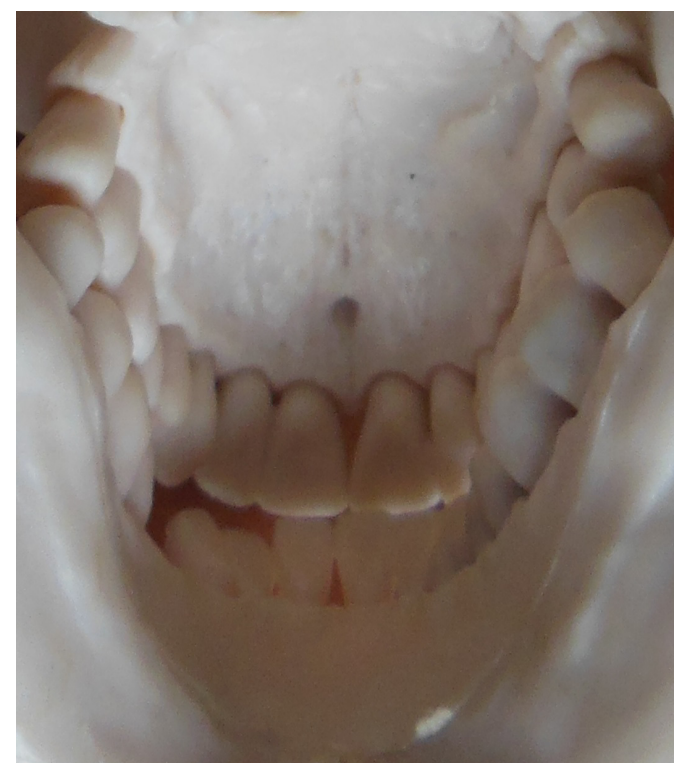

Fig. 3. (Color online) Dental image data by an intraoral scanner and 3D printing by Stereolithography Apparatus (SLA). 


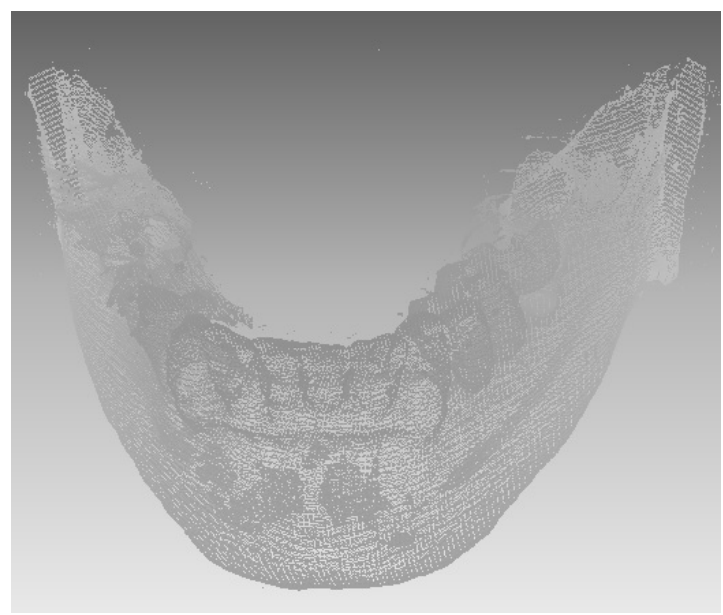

Fig. 4. Dental reconstruction modeling from CT DICOM data by Mimics ${ }^{\circledR}$ 10, software developed by Materialise Co., Ltd.

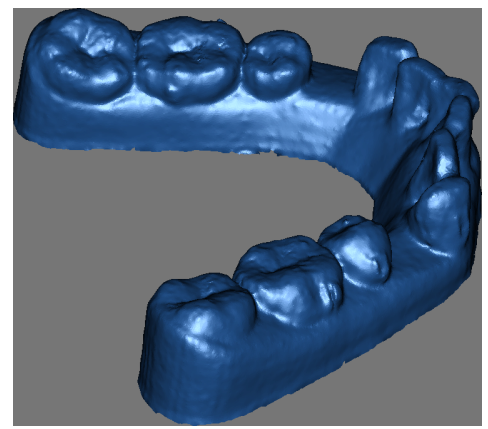

(a)

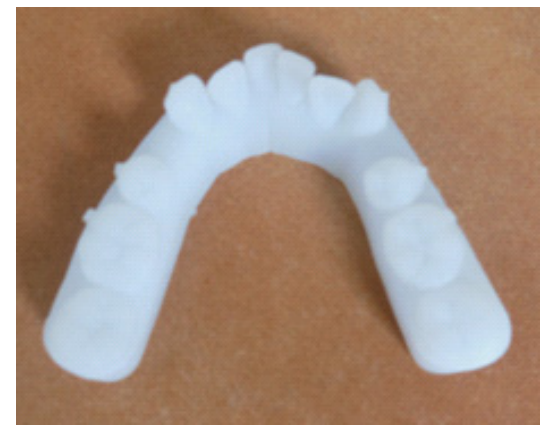

(b)

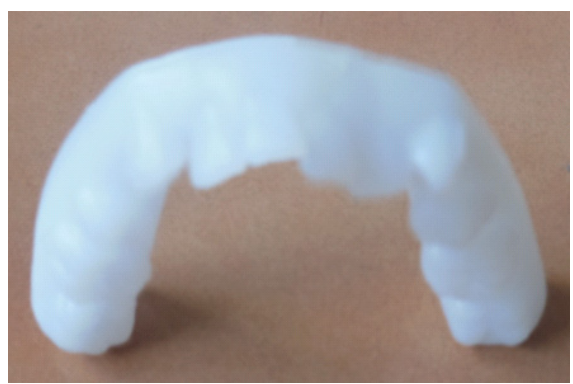

(c)

Fig. 5. (Color online) Intraoral scanning and SLA printing: (a) STL data from intraoral scanning, (b) SLA printing top view and (c) SLA printing front view. 


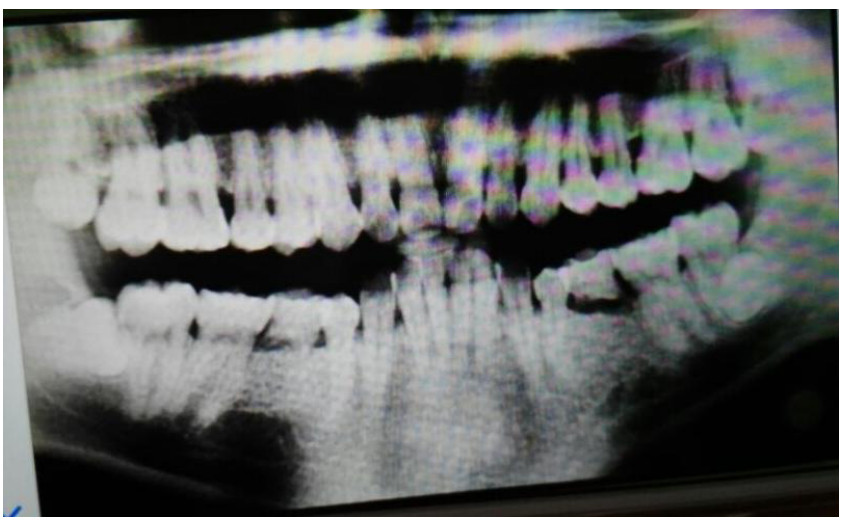

Fig. 6. Dental x-ray photo.

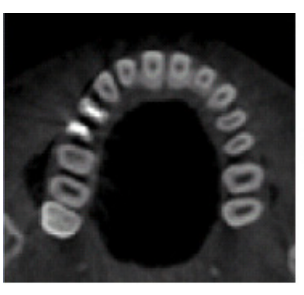

(a)

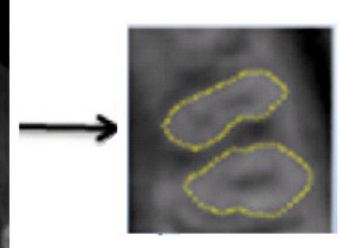

(b)

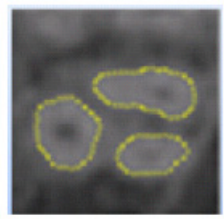

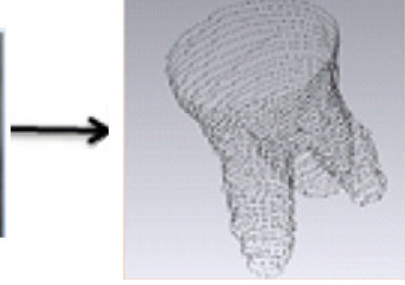

(c)

Fig. 7. Dental root 3D reconstruction by $\mathrm{CT}^{3}$ : (a) DICOM, (b) contour detection and (c) root data. Source: Hong-Tzong Yau, etc.

X-rays absorb by patients. 3D design and modeling rebuilding uses Mimics ${ }^{\circledR}$ software developed from Materialise Co., Ltd. to create a 3D printing model in the STL format.

Cone beam CT has recently been developed for dental applications. In this method, dental and maxillofacial features are scanned to reduce patient radiation exposure.

Figure 7 shows the use of DICOM data to acquire tooth root data and generate the root cloudy data by $\mathrm{CT}$ scanning. ${ }^{3}$

\section{Dental Prosthetic Design And Assembly}

Dental implants consist of a crown, fixture, abutment, and screw. ${ }^{17,18}$ The tooth crown is set on the dental fixture by a screw, as seen in Fig. 8. The dental prosthetic components are designed with CAD software, and the CAD software performs virtual assembly.

The root implant is inserted directly into the alveolar bone. The abutment connects the root implant to the crown or denture, the abutment screw connects the 


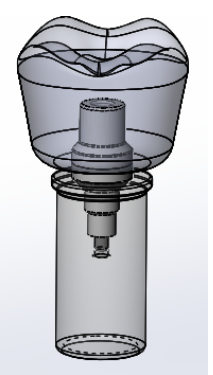

(a)

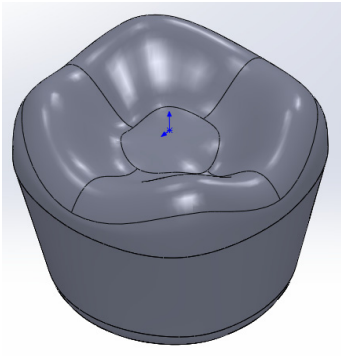

(b)

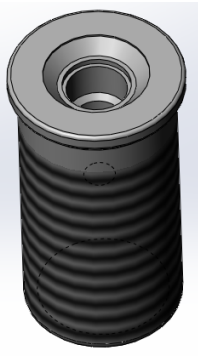

(c)

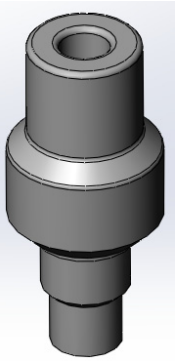

(d)

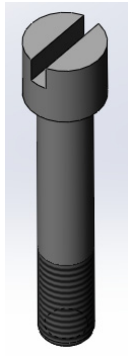

(e)

Fig. 8. Assembly drawing of dental implants: (a) Dental implant design by Solidworks, (b) dental crown, (c) fixture, (d) abutment and (e) screw.

abutment with the crown, and the crown is the dental for occlusal part. In AM fabrication, the root form, abutment, and abutment screw are considered to be printed with titanium alloy, and the crown is created with dental porcelain or titanium printing.

In Fig. 8, the internal screw attachment allows for an abutment to be frictionseated on the implant fixture. ${ }^{19}$ The implant abutments can be fabricated through the traditional $\mathrm{CAD} / \mathrm{CAM}$, or $\mathrm{AM}$ methods, ${ }^{20,21}$ or $\mathrm{AM}$ combining with $\mathrm{CAD} /$ CAM method where the plate base is printed with SLM and the fine fabrication is with tradition CAD/CAM method. ${ }^{22}$

\section{Dental Implant Printing with SLM via AM}

The dental implant root fixture is embedded in the maxilla and mandible bone for fixing the tooth crown and denture. The dental implant is placed into the jaw bone via surgery, where it obtains tissue for the retention of the solid support in the jaw bone, and connects to the tooth crown through the abutment.

Dental implants are usually fabricated out of biocompatible metal or ceramic materials. The most commonly used kinds of steel for dental implant fabrication are 316L stainless steel, CoCr alloy steel, and titanium aluminum vanadium alloy (Ti-6Al-4V).

CAM is the traditional fabrication method for dental implants. ${ }^{23}$ However, AM technology is becoming increasingly prominent and has been used in biomedical area widely, because it can print the dental crown, dental bridge, and removable partial denture.

The crown, fixture, abutment, and screw are typically fabricated out of titanium material alloy by SLM/AM technology for biocompatibility, as shown in Fig. 9. The crown can be printed with ceramic material to increase wear resistance.

Figure 10 shows tooth denture prints via an EOS GmbH SLM machine. ${ }^{24}$ 


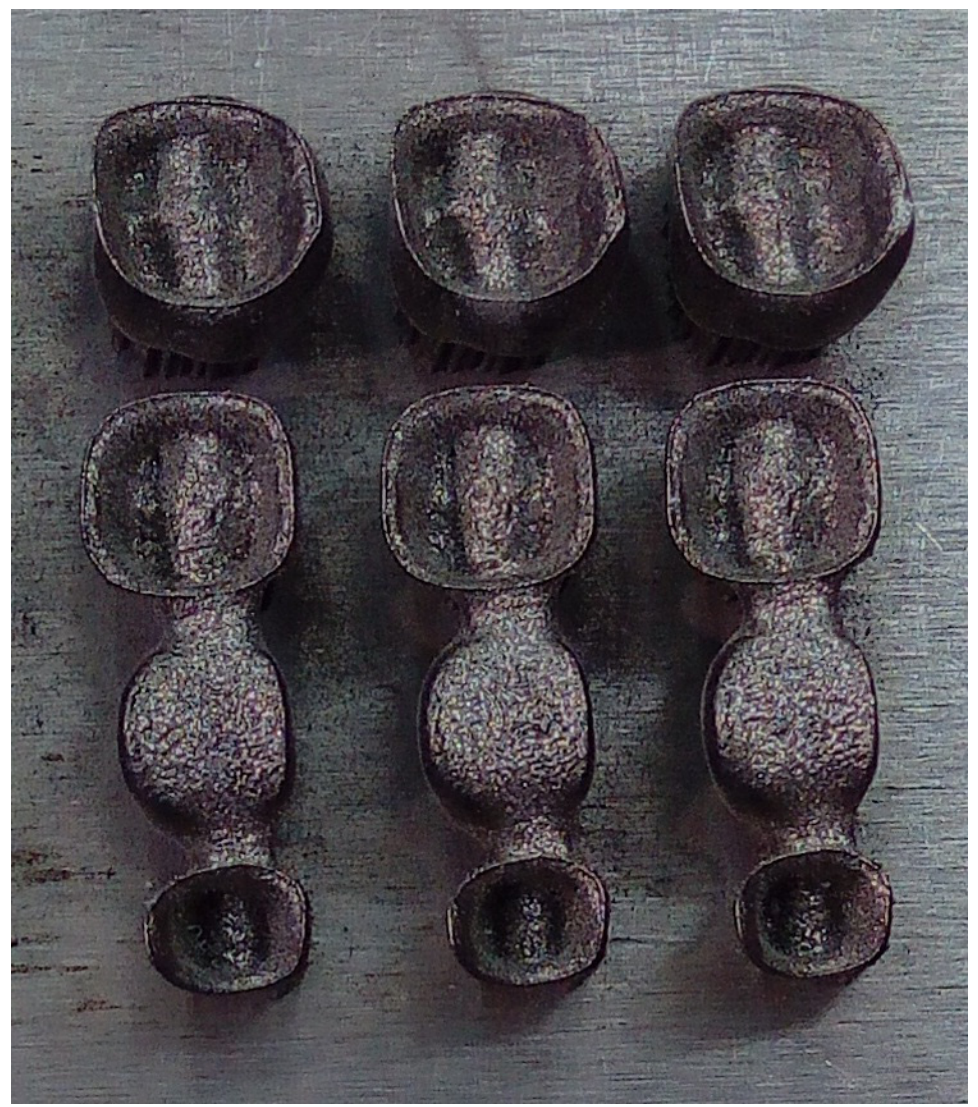

Fig. 9. (Color online) Crown and dental bridge printed via SLA with titanium alloy.

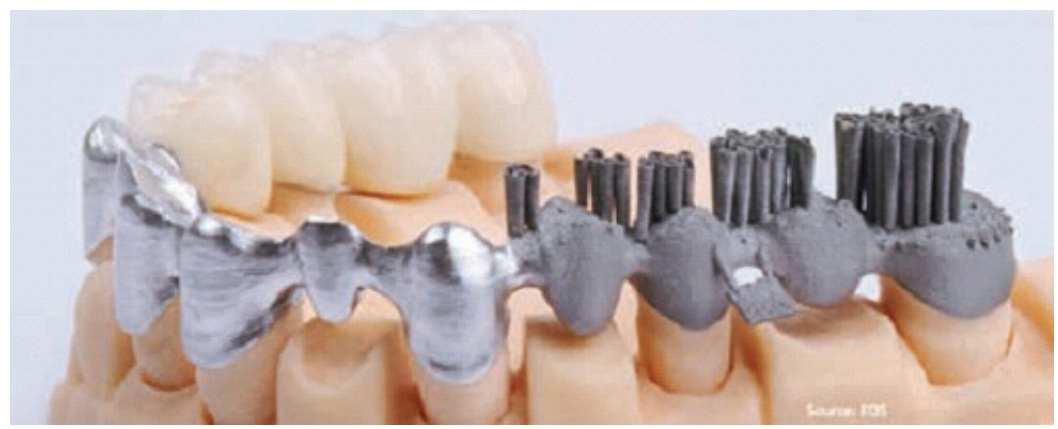

Fig. 10. (Color online) Printed dental bridge.

Source: Frank Alifui-Sebaya et al. ${ }^{24}$ 


\section{Conclusions}

This research presents a dental 3D reconstruction method by reverse engineering technology, where the dental root data is acquired by CT DICOM data from CT scanning, and the tooth crown model is built by cloudy point data from impression model scanning, or the intraoral scanner. The research compares the dental implant traditional fabricating method to SLM/AM technology, and it shows that a customized dental implant fabricated via SLM/AM has adequate dimension accuracy.

\section{Acknowledgments}

This work was financially supported by National Natural Science Foundation of China (11472200).

We would like to thank the Top Health Medical Management (THM) Co., in Guangzhou, China, for conducting the intraoral scanning.

\section{References}

1. Martorelli M, Gerbino S, Giudice M, Ausiello P, A comparison between customized clear and removable orthodontic appliances manufactured using RP and CNC techniques, Dent Mater 29:e1-e10, 2013.

2. van Noort R, The future of dental devices is digital, Dent Mater 28:3-12, 2012.

3. Yau HT, Yang TJ, Chen YC, Tooth model reconstruction based upon data fusion for orthodontic treatment simulation, Comput Biol Med 48:8-16, 2014.

4. Chen J, Zhang Z, Chen X, Zhang C, Zhang G, Xu Z, Design and manufacture of customized dental implants by using reverse engineering and selective laser melting technology, J Prosthet Dent 112(5):1088-1095, 2014.

5. Lee SJ, Gallucci GO, Digital vs conventional implant impressions: Efficiency outcomes, Clin Oral Implants Res 24:111-115, 2013.

6. Lin WS, Harris BT, Zandinejad A, Morton D, Use of digital data acquisition and CAD/ CAM technology for the fabrication of a fixed complete dental prosthesis on dental implants, J Prosthet Dent 111:1-5, 2014.

7. Lin WS, Chou JC, Metz MJ, Harris BT, Morton D, Use of intraoral digital scanning for a CAD/CAM-fabricated milled bar and superstructure framework for an implant-supported, removable complete dental prosthesis, J Prosthet Dent 113:509-515, 2015.

8. Wang K, The use of titanium for medical applications in the USA, Mater Sci Eng A 213:134-137, 1996.

9. Kannan MB, Raman RK, In vitro degradation and mechanical integrity of calciumcontaining magnesium alloys in modified-simulated body fluid, Biomaterials 29:23062314, 2008.

10. Hench LL, Wilson J, Surface-active biomaterials, Science 226(4675):630-636, 1984.

11. Peterson L, McKinney C, Pennel B, Klawitter J, Weinstein A, Clinical, radiographical, and histological evaluation of porous rooted cobalt-chromium alloy dental implants, J Dent Res 59:99, 1980.

12. Cook, SD, Klawitter JJ, Weinstein AM, A model of the implant-bone interface, characteristics of Porous Dental Implants, J Dent Res 61(8):1006-1009, 1982.

13. Lin WS, Harris BT, Zandinejad A, Morton D, Use of digital data acquisition and CAD/ CAM technology for the fabrication of a fixed complete dental prosthesis on dental implants, J Prosthet Dent 111(1):1-5, 2014. 
14. Zhang L, Morsi Y, Wang Y, Li Y, Ramakrishna S, Review scaffold design and stem cells for tooth regeneration, Jpn Dent Sci Rev 49:14-26, 2013.

15. Nishii Y, Nojima K, Takane Y, Isshiki Y, Integration of the maxillofacial three-dimensional CT image and the three-dimensional dental surface image, The Journal of Japan Orthodontic Society 57:189-194, 1998.

16. Parks ET, Williamson GF, Digital radiography: An overview, J Contemp Dent Pract 3(4):23-39, 2002.

17. Terai H, Shimahara M, Sakinaka Y, Tajima S, Accuracy of integration of dental casts in three-dimensional models, J Oral Maxillofacial Surgery 57:662-665, 1999.

18. Bahrami B, Shahrbaf S, Mirzakouchaki B, Ghalichi F, Ashtiani M, Martin N, Effect of surface treatment on stress distribution in immediately loaded dental implants-A 3D finite element analysis, Dent Mater 30:e89-e97, 2014.

19. Lee JH, Frias V, Lee KW, Wright RF, Effect of implant size and shape on implant success rates: A literature review, 94(4):377-381, 2005.

20. Protopapadaki M, Monaco EA Jr, Kim HI, Davis EL, Comparison of fracture resistance of pressable metal ceramic custom implant abutment with a commercially fabricated CAD/CAM zirconia implant abutment, J Prosthet Dent 110(5):389-96, 2013.

21. Bertolini MD, Kempen J, Louren EJ, Telles DD, The use of CAD/CAM technology to fabricate a custom ceramic implant abutment: A clinical report, J Prosthet Dent 3(5):362-366, 2014.

22. Jiang BG, Xianghui W, Tan ZH, Fabricating titanium denture base plate by laser rapid forming, Rapid Prototyping J 15(2):133-136, 2009.

23. Bertolini Mde M, Kempen J, Lourenço EJ, Telles Dde M, The use of CAD/CAM technology to fabricate a custom ceramic implant abutment: A clinical report, J Prosthet Dent 111:362-366, 2014.

24. Alifui-Sebaya F, Evans J, Eggbeer D, George R, Clinical relevance of Laster-Sintered CO-CR alloys for prosthodontic treatments: A review, Proc Int Conf on Progress in Additive Manufacturing, pp. 115-120, 2014. 